\title{
Can renal ultrasonography and DMSA scintigraphy be used for the prediction of irreversible histological lesions of the upper pole in duplex system with ureteroceles or ectopic ureters?
}

\author{
Oldrich Smakala, Jan Sarapatkaa, Igor Hartmann ${ }^{\mathrm{a}}$, Ondrej Vencalek ${ }^{\mathrm{b}}$, Hana Flogelovac , Pavel Korandad ${ }^{\mathrm{d}}$, Vladimir Student ${ }^{\mathrm{a}}$
}

\begin{abstract}
Aim. To assess of the role of renal ultrasonography (US) and DMSA renal scintigraphy in the prediction of irreversible histological lesions of the upper pole in duplex system.

Methods. A prospective cohort study based on data collected between 2005 and 2012 at our institution. The cohort consisted of 23 patients with ureteroceles and 28 patients with ectopic ureters who underwent upper pole nephrectomy. Preoperative recordings from ultrasound and nuclear renal scans were compared with the histological findings. Histological irreversible lesions were defined as the presence of dysplasia and/or severe chronic interstitial nephritis (CIN) in $\geq 90 \%$ of the specimen. ROC (Receiver Operating Characteristic) curves were used to investigate thresholds in order to identify irreversible lesions using various differential functions. The histology was correlated with the results of imaging.

Results. Pathological findings were found in all histological samples. Histological lesions were irreversible in 20/23 patients $(87.0 \%)$ with ureteroceles and in $14 / 28$ patients (50.0\%) with ectopic ureters. The model is able to predict irreversible lesions if an upper pole differential function is $\leq 3 \%$ in patients with ureteroceles, and $\leq 2 \%$ in the presence of ectopic ureters. Weak association between parenchymal thinning on ultrasonography and irreversible lesions was found in patients with ectopic ureters.

Conclusion. DMSA renal scintigraphy provides a useful tool for the prediction of irreversible lesions in the upper pole. Low differential function ( $\leq 3 \%$ and $\leq 2 \%$, respectively) indicates irreversible lesions, favoring heminephrectomy. Higher differential function indicates greater remaining biological potential of the parenchyma, favoring reconstruction of the upper pole.
\end{abstract}

Key words: ectopic ureter, ureterocele, duplex kidney, ultrasonography, renal scintigraphy

Received: January 24, 2016; Accepted: April 27, 2016; Available online: May 12, 2016

http://dx.doi.org/10.5507/bp.2016.028

${ }^{a}$ Department of Urology, University Hospital Olomouc, Czech Republic

${ }^{b}$ Department of Mathematical Analysis and Applications of Mathematics, Faculty of Science, Palacky University Olomouc, Czech Republic 'Department of Paediatrics, University Hospital Olomouc, Czech Republic

${ }^{d}$ Department of Nuclear Medicine, University Hospital Olomouc, Czech Republic

Corresponding author: Jan Sarapatka, e-mail: h.sarapatka@seznam.cz

\section{INTRODUCTION}

The choice of proper medical treatment of ureteroceles or ectopic ureters in the duplex kidney remains a challenge despite the availability of several studies addressing this problem ${ }^{1-6}$. In current practice, treatment is based on morphological information from ultrasonography (US), information about function from the dimercaptosuccinic acid (DMSA) or mercaptoacetyltriglycine (MAG-3) renal scan, and the clinical course. However, due to the lack of objective criteria for evaluation of the obtained patients' records, the decision-making is largely empirical. The choice between reconstructive and ablative surgery may be facilitated by knowledge of the biological potential of the upper pole's parenchyma.

The aim of this study is, 1- to assess the possible use of US and DMSA renal scintigraphy for preoperative prediction of the most severe - irreversible histological lesions of the upper pole (UP) in patients with ureteroceles or ectopic ureters in duplex systems, 2- to find a threshold of UP differential function measured by nuclear renal scans in order to define the group of patients with an UP with little or no potential for recovery, 3- to examine the power of association between histological lesions in the UP and three characteristics gained by renal US, namely echogenicity, parenchymal thinning and degree of hydronephrosis.

\section{PATIENTS AND METHODS}

A cohort study was performed on patients with ectopic ureters or ureteroceles in the duplex system who underwent upper pole partial nephrectomy (UPN) at our institution between January 2006 and November 2013. During this period, 57 patients were diagnosed with ureteroceles in the duplex system, and 36 patients were diagnosed with ectopic ureters in the duplex system. Of these, 23 patients with ureteroceles and 28 patients with ectopic ureters underwent UPN. The rest underwent reconstruc- 
tive surgery. All patients underwent a preoperative workup including renal US and DMSA nuclear renal scans.

Three characteristics obtained by US were recorded: degree of hydronephrosis, parenchymal thinning and increased echogenicity. The degree of hydronephrosis was characterized by anteroposterior renal pelvic diameter (AP) of the UP. Three categories were distinguished: an AP less than $10 \mathrm{~mm}$, an AP of 10-15 mm and an AP of more than $15 \mathrm{~mm}$. Parenchymal thickness measured at the upper calyx ranged from 1 to $5 \mathrm{~mm}$ constituting five categories of parenchymal thickness. The affected UP was evaluated for echogenicity compared to the liver. Two categories of echogenicity were distinguished: increased and normal.

Tc-99m DMSA scintigraphy was used to assess function of the affected UP. Differential function of the UP - (the percentage of overall function measured by renal uptake) was determined using the high-resolution images. Demarcation of the renal segments of the duplex system was determined on visual inspection of the images (Fig. 1). In order to refine determination of the extent of upper and lower segments in a duplex kidney, measurement of the dimensions of both segments from US findings was used (Fig. 2).
UPN specimens were fixed in formalin and stained with hematoxylin and eosin, Masson's trichrome and periodic acid-Schiff (PAS). Histological sections of renal specimens were evaluated by one pathologist. The histological lesions were classified, according to the glomerular and tubulointerstitial alterations and presence of dysplasia, into four groups. The normal group included mature renal parenchyma with only few lesions. The mild chronic interstitial nephritis (CIN) group included more-prominent glomerular sclerosis as well as mild interstitial fibrosis and tubular atrophy. The severe CIN group included cystic changes with parenchymal loss, glomerulosclerosis, extensive interstitial fibrosis and tubular atrophy ${ }^{7}$. The dysplasia group was characterized by the persistence of immature and incompletely differentiated renal tissue ${ }^{8}$. In each specimen the percentage presence of individual levels of histological change and dysplasia was evaluated. From all examinations, the average afflicted area of each specimen, by individual types of histological changes, was calculated. Irreversible histological lesions were defined as presence of dysplasia and/or severe CIN in at least $90 \%$ of the specimen. All patients with ureteroceles underwent initial endoscopic decompression. The indications for surgery included a persisting poor function of the upper renal
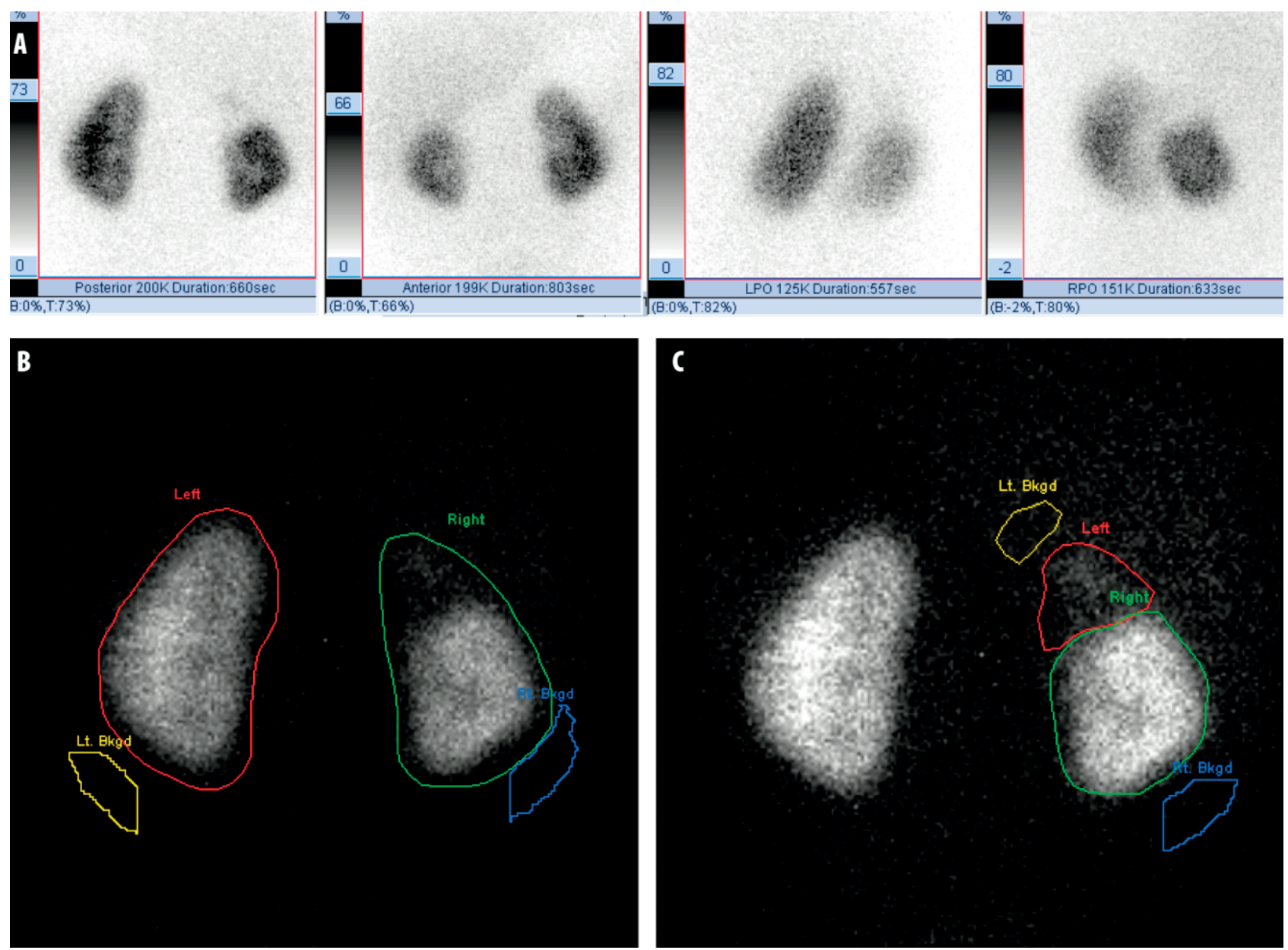

Fig. 1. Tc-99m DMSA scintigraphy.

Regions of interest, normal parenchymal accumulation of lower pole, poor visualisation of upper pole (differential function 7\%), no lesions detected. 


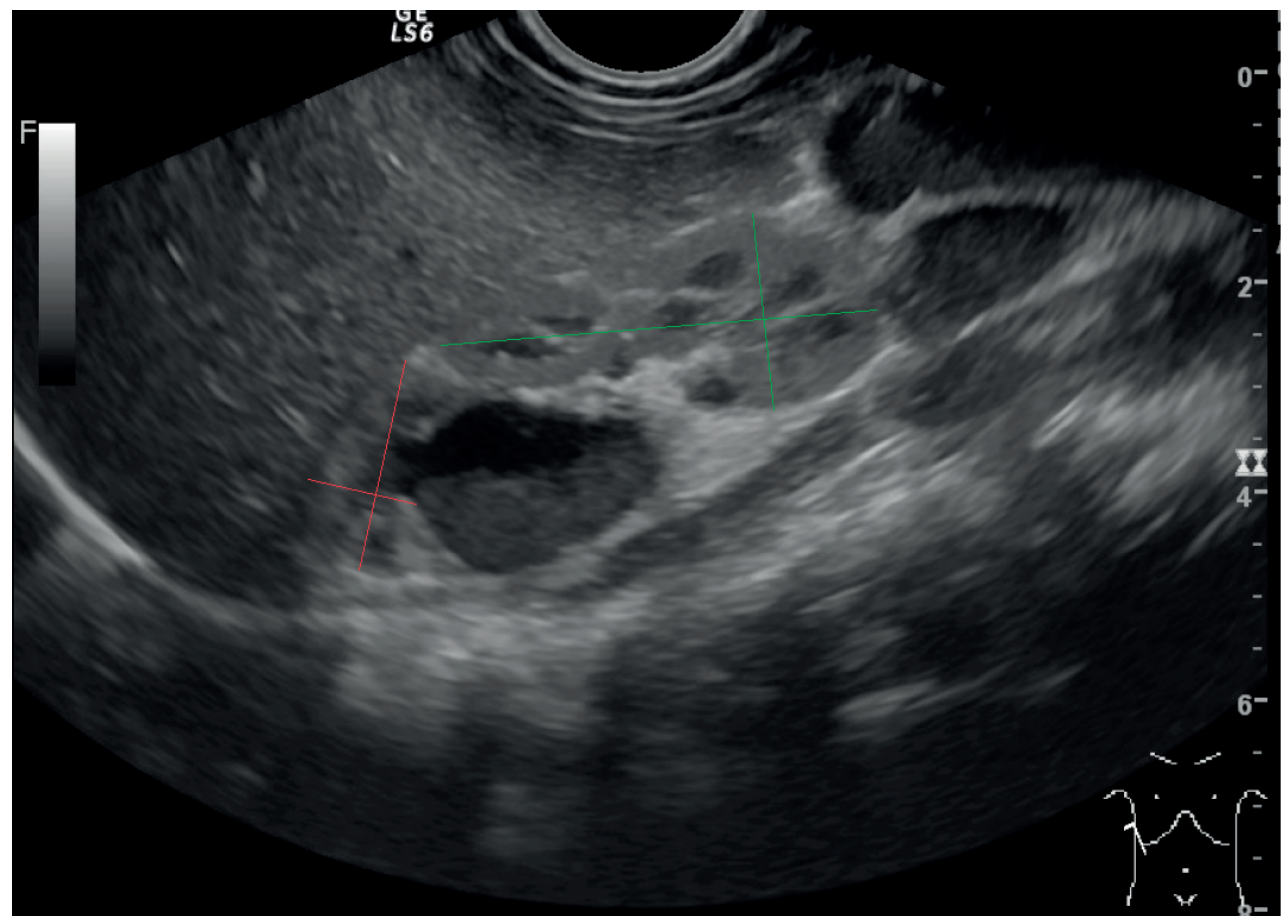

Fig. 2. Ultrasound image of the same patient.

Duplex system with dilated UP renal pelvis. Measurement of upper and lower segments was performed and compared with scintigraphy findings in order to refine the determination of the extent of both segments.

segment (less than $10 \%$ of the overall renal function) and/ or recurrent urinary tract infection and severe hydronephrosis. Parents' choice of treatment was respected.

All statistical analyses were performed separately for ureteroceles and ectopic ureters.

Power of association between patients UP lesions and any of their characteristics from US was described by correlation coefficient ${ }^{9}$. The uncertainty of the correlation coefficient estimation was described by the bootstrap $95 \%$ confidence interval ${ }^{10}$.

The predictive value of differential function of the upper poles was investigated in detail. The patients could be divided into two subgroups according to their UP differential function. All possible thresholds dividing the group of patients into subgroups with low and high differential function of the UP were examined. Each threshold implied a certain sensitivity and specificity. The quality of predictions under various thresholds was displayed by ROC curves ${ }^{11}$. The uncertainty in construction of ROC curves was visualized by confidence bands for the ROC curves. The method of vertical averaging was used for the construction of these $95 \%$ confidence bands ${ }^{12}$. The total predictive value of differential function of the UP was described by the area under the ROC curve.

\section{RESULTS}

\section{Ureteroceles}

Fifty seven patients with ureteroceles underwent surgery ( 29 girls, 28 boys). In 43 cases ( $75 \%$ ) the ureterocele was diagnosed antenatally, in 14 (25\%) cases the diag- nosis was based on clinical symptoms. Reconstruction was performed in 34 cases. UPN was performed in 23 (40\%) cases ( 14 girls, 9 boys). In 15 (65\%) cases where UPN was performed the ureterocele was diagnosed antenatally. Median age at sugery was 19.4 months (range $3-80)$. Irreversible lesions were found in 20 of 23 patients $(87.0 \%)$ with ureteroceles; 10 of 23 patients (43.5\%) had dysplasia in their upper poles (Table 1).

The results from US were available for all 23 patients who underwent UPN. AP was more than $15 \mathrm{~mm}$ in 14 (61\%) cases, $10-15 \mathrm{~mm}$ in seven $(30 \%)$ cases and less than $10 \mathrm{~mm}$ in two (9\%) cases. Increased echogenicity was found in nine patients (39\%). Parenchyma thickness ranged from 1 to $5 \mathrm{~mm}$ which was more than $50 \%$ reduction with respect to the contralateral kidney in all patients. Correlation coefficients between irreversible histological lesions and individual US characteristics were estimated to be 0.046 (interval $-0.371-0.393$ ) for increased echogenicity, 0.064 (interval $-0.387-0.535$ ) for parenchyma reduction and for the degree of hydronephrosis 0.112 (interval -0.368-0.656). None of the evaluated US characteristics was associated with irreversible microscopic lesions in UP parenchyma.

Renal scans were available for all 23 patients who underwent UPN. Eleven (46\%) patients had nonfunctioning UP, maximal differential function was $5 \%$ (in one patient). The median was $1 \%$ (Table 1 ). The main aim of the study was to predict irreversible histological lesions of UP from its differential function. Fig. 3 displays the ROC curve illustrating the quality of predictions under various thresholds. The area under the ROC curve is equal to 0.617 , with the $95 \% \mathrm{CI}=(0.225,1.00)$. The $90 \%$ sensitivity is reached 
for the threshold equal to $3 \%$. Thus the model predicts irreversible lesions in patients with the UP differential function equal to $3 \%$ or less. The group of patients with function not higher than $3 \%$ constitutes about $91 \%$ of the patients with ureteroceles who underwent UPN (21 of 23 patients). The model is characterized by $95 \%$ sensitivity, $\mathrm{CI}=(84 \%, 100 \%)$ and $33 \%$ specificity, $\mathrm{CI}=(0 \%, 100 \%)$. The total accuracy is $87 \%, \mathrm{CI}=(74 \%, 100 \%)$. Evaluation by bootstrap has shown that the threshold equal to $3 \%$ would result in $90 \%$ sensitivity with a specificity of about $87 \%$.
Table 1. Number of patients with ureteroceles or ectopic ureters according to the upper pole function and severity of the upper pole histological lesions.

\begin{tabular}{ccccc}
\hline & \multicolumn{2}{c}{ Ureteroceles } & \multicolumn{2}{c}{ EctopicUreters } \\
Function & Irreversible & Other & Irreversible & Other \\
\hline 0.00 & 10 & 1 & 5 & 1 \\
0.01 & 5 & 1 & 5 & 0 \\
0.02 & 1 & 0 & 3 & 2 \\
0.03 & 3 & 0 & 1 & 2 \\
0.04 & 0 & 0 & 0 & 1 \\
0.05 & 1 & 1 & 0 & 1 \\
0.06 & 0 & 0 & 0 & 1 \\
$>0.06$ & 0 & 0 & 0 & 6 \\
Total & 20 & 3 & 14 & 14 \\
\hline
\end{tabular}

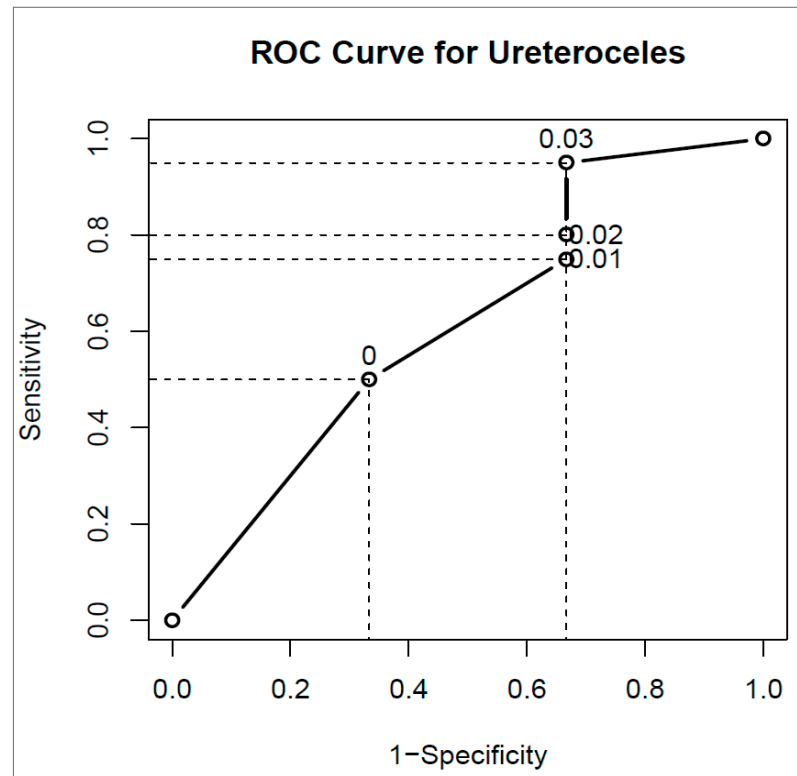

95\% Confidence bands for the ROC Curve for Ureteroceles

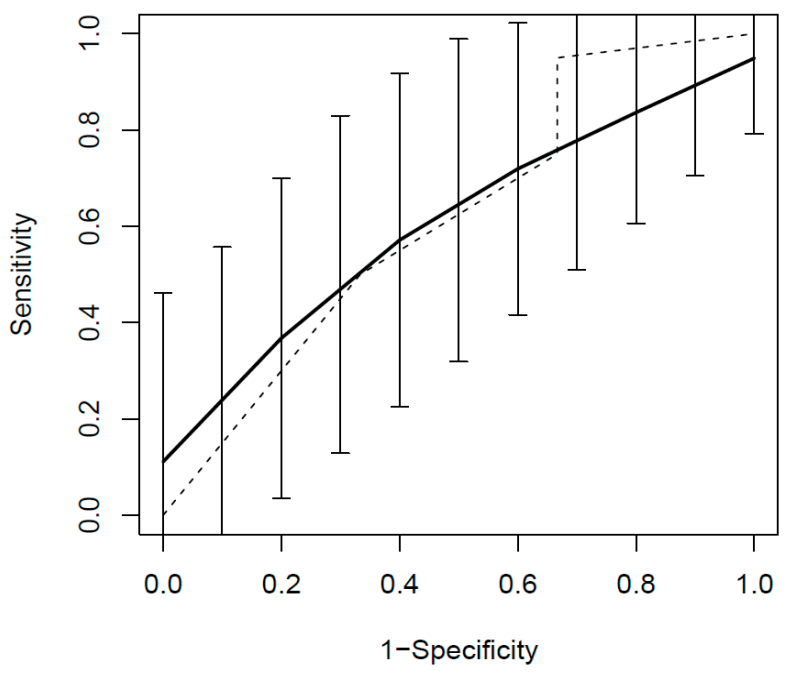

Fig. 3. ROC curve illustrating the quality of predictions under various thresholds. The model predicts irreversible histological lesions in patients with the UP differential function equal to $3 \%$ or less.

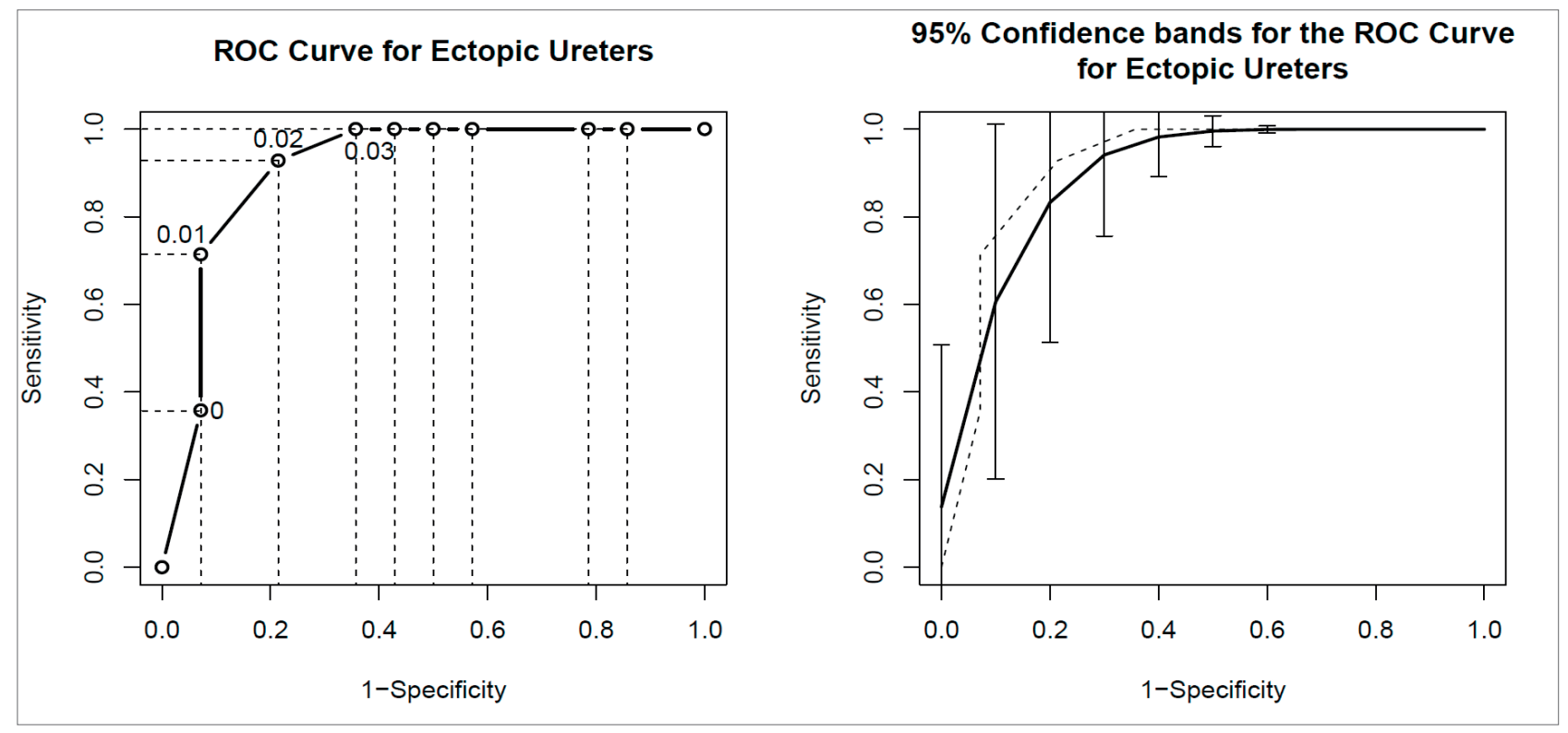

Fig. 4. ROC curve illustrating the quality of predictions under various thresholds. The model predicts irreversible histological lesions in patients with the UP differential function equal to $2 \%$ or less. 


\section{Ectopic ureters}

Thirty six patients with ectopic ureters underwent surgery ( 26 girls, 10 boys). In 30 cases ( $83 \%$ ) the ectopic ureter was diagnosed antenatally, in $6(17 \%)$ cases the diagnosis was based on clinical symptoms. Reconstruction was performed in 8 cases. UPN was performed in 28 (78\%) cases (21 girls, 7 boys). In 18 cases (64\%) where UPN was performed the ectopic ureter was diagnosed antenatally. Median age at surgery was 17.1 months (range 3-77).

Histological lesions were irreversible in 14 of 28 patients $(50 \%)$ with ectopic ureters; 2 of 28 patients $(7.1 \%)$ had dysplasia in their upper poles. More detailed information is included in Table 1.

The results from US were available for all 28 patients who underwent UPN. AP was more than $15 \mathrm{~mm}$ in $17(61 \%)$ cases and $10-15 \mathrm{~mm}$ in $11(39 \%)$ cases. No patient had AP less than $10 \mathrm{~mm}$. Increased echogenicity was found in 9 patients (32\%). Parenchyma thickness ranged from 1 to $5 \mathrm{~mm}$, that means more than $50 \%$ reduction with respect to the contralateral kidney, in 27 of 28 patients (96\%). Correlation coefficients between irreversible histological lesions and individual US characteristics were estimated to be 0.149 (interval $-0.220-0.500$ ) for increased echogenicity, and 0.073 (interval -0.316-0.456) for the degree of hydronephrosis. Neither increased echogenicity nor degree of hydronephrosis was associated with severity of histological lesions in UP.

The correlation coefficient between irreversible histological lesions and parenchymal reduction is estimated to be -0.346 with $95 \% \mathrm{CI}=(0.673,0.024)$. Lower values of parenchymal thickness thus indicate higher probability of irreversible lesions in UP. The association between parenchymal reduction and severity of lesions is not too strong, but it appears to be statistically significant with a significance level of slightly more than $5 \%$.

Renal scans were available for all 28 patients who underwent UPN. Six (21\%) patients had nonfunctioning UP, maximal differential function was $9 \%$ (in two patients). The median was $2 \%$. Detailed information is shown in Table 1.

The possibility to predict irreversible histological lesions of the UP from its differential function was investigated. Fig. 4 displays the ROC curve illustrating the quality of predictions under various thresholds. The area under the ROC curve is equal to 0.911 , with the $95 \% \mathrm{CI}=$ $(0.763,0.997)$. The model predicts irreversible histological lesions of the UP in patients with UP function equal to $2 \%$ or less. The group of patients with function not higher than $2 \%$ constitutes about $57 \%$ of the patients with ectopic ureters who underwent UPN (16 of 28 patients). Total accuracy of this prediction - percentage of correctly classified patients - is about $85.7 \%(71.4 \%, 96.4 \%)$. The model is characterized by $93 \%$ sensitivity, $\mathrm{CI}=(76 \%, 100 \%)$, and $79 \%$ specificity, $\mathrm{CI}=(55 \%, 100 \%)$.

\section{DISCUSSION}

There are many studies dealing with evaluation of histological findings in UP specimens after UPN in the case of ureteroceles or ectopic ureters in duplex kidney ${ }^{1,3,4,13-15}$. However, only little attention has been dedicated to the predictive value of preoperatively collected records for the presence and severity of histological lesions in this situation $^{16-18}$.

Faure et al. ${ }^{18}$ proved that severe thinning on US and minimal pole function on MRU can be used to predict the severity of histological lesions in duplex systems.

In their study Meneghesso et al. ${ }^{17}$ did not prove the possibility of predicting the severity of histological lesions of the UP in the case of ureteroceles or ectopic ureters in duplex kidney by renal US or MAG-3 renal scintigraphy.

Bolduc et al. ${ }^{16}$ compared the diagnostic imaging findings with the histological lesions in UPN specimens of duplex system ureteroceles, using renal US and DMSA or MAG-3 nuclear renal scans. It was proved that severe parenchymal thinning on renal US and minimal function ( $\leq 4 \%$ overall renal function) on nuclear renal scan can be used to predict the severity of histological lesions of the UP.

Our study is consistent with previously published data ${ }^{16,18}$ which suggest that there is no association between histological lesions in UP and echogenicity or degree of hydronephrosis. Our study confirms the results of Bolduc et al. and Faure et al. ${ }^{16,18}$ which indicated that severe parenchyma reduction is associated with a severe histological lesion. We found this association in patients with ectopic ureter. The association is not strong enough but the level of parenchymal reduction might be used as a subsidiary marker of irreversible lesions in UP.

Like Bodduc, we believe that the differential function of UP measured preoperatively on DMSA renal scans can be used to predict the severity of histological lesions in the UP. We have been successful in determining the threshold value for UP function defining the seriousness of histological change in parenchyma. In patients with ureterocele a threshold value of $3 \%$ was set, while for patients with an ectopic ureter a $2 \%$ threshold value was defined. Patients with function below the threshold values have a high probability of the most severe, irreversible histological change. Both suggested thresholds are lower than the artificially designated thresholds presented in Bolduc et al.'s studies ${ }^{16}$. We believe that this reduction is influenced by two factors as follows:

1) Bolduc defined a $25 \%$ presence of TIN or dysplasia as the threshold for serious damage to parenchyma. We have interpreted $90 \%$ affliction of parenchyma as an irreversible change in kidney tissue. This is based on the pathologist's view that $90 \%$ evidence of chronic TIN or dysplasia in kidney parenchyma is the terminal phase of damage to kidney parenchyma with no chance of regeneration.

2) We have refined the statistical evaluation of results. Bolduc et al. set a 4\% threshold value arbitrarily, and used it to test the statistical significance of series without prior 
knowledge of a zero hypothesis. We believe that our approach, of seeking threshold values by means of a ROC curve, is more accurate.

From our study it is apparent that the most serious damage to UP kidney parenchyma is in a state close to afunctional and the level of damage is more serious in the case of ureterocele than in ectopic ureter. However the validity of this hypothesis, that the ureteroceles lead to more severe damage, is not clear and should be further investigated.

Definition of threshold values for UP function in combination with irreversibly damaged parenchyma replaces the descriptive notion of poor UP function with exact numerical data. Setting threshold levels may contribute to a reduction in the number of ablation procedures (heminephrectomy) and reconstruction operations being carried out more often, even in the case of considerable UP duplex kidney hypofunction in ectopic ureter or ureterocele. Long-term ultrasound and radionuclide monitoring of patients in combination with careful evaluation of clinical progress will verify the correctness of the chosen procedure.

\section{CONCLUSION}

DMSA renal scintigraphy provides a useful tool for the prediction of irreversible lesions in the UP. Low differential function ( $3 \%$ or less in the case of ureteroceles, $2 \%$ or less respectively in the case of ectopic ureters) indicates irreversible lesions, favoring management by heminephrectomy. Higher differential function indicates higher remaining biological potential of the parenchyma, favoring reconstructive surgery. Ultrasonography plays a crucial role in the antenatal diagnosis of ectopic ureters and ureteroceles, however its role in the prediction of the severity of histological lesions in the UP is minimal.

\section{ABBREVIATIONS}

AP, Anteroposterior renal pelvic diameter; AUC, Area under curve; CIN, Chronic interstitial nephritis; DMSA, Dimercaptosuccinic acid; MAG-3, Mercaptoacetyltriglycine; ROC, Receiver Operating Characteristic; UP, Upper pole; UPN, Upper pole partial nephrectomy; US, Ultrasonography.

Author contributions: OS, JS, IH, HF, PK: data acquisition, OS, JS: drafting the manuscript, OV: statistical analysis, IH, HF, OV, PK, VS: critical revision.

Conflict of interest statement: None declared.

\section{REFERENCES}

1. Vates TS, Bukowski T, Triest J, Freedman A, Smith C, Perlmutter A, Gonzalez R. Is there a best alternative to treating the obstructed upper pole? J Urol 1996;156 (2 Pt 2):744-6.

2. Husmann DA, Ewalt DH, Glenski WJ, Bernier PA. Ureterocele associated with ureteral duplication and a nonfunctioning upper pole segment: management by partial nephroureterectomy alone. J Urol 1995;154(2 Pt 2):723-6.

3. Smith FL, Ritchie EL, Maizels M, Zaontz MR, Hsueh W, Kaplan WE, Firlit CF. Surgery for duplex kidneys with ectopic ureters: ipsilateral ureteroureterostomy versus polar nephrectomy. J Urol 1989;142 (2 Pt 2):532-4.

4. el Ghoneimi A, Miranda J, Truong T, Monfort G. Ectopic ureter with complete ureteric duplication: conservative surgical management. J Pediatr Surg 1996;31(4):467-72.

5. Gran CD, Kropp BP, Cheng EY, Kropp KA. Primary lower urinary tract reconstruction for nonfunctioning renal moieties associated with obstructing ureteroceles. J Urol 200;173(1):198-201.

6. Han MY, Gibbons MD, Belman AB, Pohl HG, Majd M, Rushton HG.: Indications for nonoperative management of ureteroceles. J Urol 2005;174(4 Pt 2):1652-5.

7. Murer L, Benetti E, Centi S, Della Vella M, Artifoni L, Capizzi A, Zucchetta P, DelPrete D, Carasi C, Montini G, Rigamonti W, Zaccello G. Clinical and molecular markers of chronic interstitial nephropathy in congenital unilateral ureteropelvic junction obstruction. J Urol 2006;176(6 Pt 1):2668-73.

8. Bolduc S, Upadhyay J, Sherman C, Farhat W, Bagli DJ, McLorie GA, Khoury AE, El-Ghoneimi A.: Histology of upper pole is unaffected by prenatal diagnosis in duplex system ureteroceles. J Urol 2002;168(3):1123-6.

9. Agresti, Alan. Analysis of Ordinal Categorical Data. Hoboken, NJ: Wiley, 2010.

10. Efron, Bradley, and Robert Tibshirani. An Introduction to the Bootstrap. New York: Chapman\&Hall, 1994.

11. Hastie, Trevor, Robert Tibshirani, and J. H. Friedman. The Elements of Statistical Learning: Data Mining, Inference, and Prediction. New York: Springer, 2009.

12. Macskassy SA, Provost FJ (2004). Confidence Bands for ROC Curves. CeDER Working Paper 02-04, Stern School of Business, New York University, NY, NY 10012. Jan 2004.

13. Abel C, Lendon M, Gough DC. Histology of the upper pole in complete urinary duplication-does it affect surgical management? $\mathrm{Br}$ J Urol 1997;80(4):663-5.

14. Arena F, Nicotina A, Cruccetti A, Centonze A, Arena S, Romeo G. Can histologic changes of the upper pole justify a conservative approach in neonatal duplex ectopic ureterocele? Pediatr Surglnt 2002;18(8):681-4.

15. Monfort G, Guys JM, Coquet M, Roth K, Louis C, Bocciardi A. Surgical management of duplex ureteroceles. J Pediatr Surg 1992;27(5):6348.

16. Bolduc S, Upadhyay J, Restrepo R, Sherman C, Farhat W, Bägli DJ, McLorie GA, Khoury AE, El Ghoneimi A. The predictive value of diagnostic imaging for histological lesions of the upper poles in duplex systems with ureteroceles. BJU Int 2003;91(7):678-82.

17. Meneghesso D, Castagnetti M, Della Vella M, Benetti E, Zucchetta P, Rigamonti W, Murer L. Clinico-pathological correlation in duplex system ectopic ureters and ureteroceles: can preoperative work-up predict renal histology? Pediatr Surglnt 2012;28(3):309-14.

18. Faure A, Merrot T, Sala Q, Chaumoitre K, Guys JM, Bourliere-Najean B, Torrents J, Mundler O, Lechevallier E, Alessandrini P. Value of diagnosis imaging in the evaluation of the severity of histological lesions in duplex systems. J Pediatr Urol 2014;10(2):361-7. 